\title{
Dinámica del mercado laboral del sector transporte en México
}

\author{
Dynamics of the labor market of the transport sector in Mexico \\ Humberto Ríos Bolivar * y Gabriela Cruz González
}

Instituto Politécnico Nacional, México

Recibido el 24 de Septiembre de 2016; aceptado el 09 de Octubre de 2017

Disponible en Internet el 7 de diciembre de 2018

\section{Resumen}

En el presente estudio se analiza la dinámica y la estructura del sector laboral del transporte en México, específicamente de los "Conductores de transporte y de maquinaria móvil", así como el impacto del crecimiento económico sobre la demanda de trabajo por categorías de los trabajadores de dicho sector de acuerdo a la Clasificación Única de Ocupaciones del sector laboral mexicano. Se utiliza la metodología presentada por Kato, E. (2004) y siguiendo a Sydsaeter, K. y Hammond, P. (1995), para determinar la elasticidad producto del empleo para el periodo 1996-2012, haciendo uso de microdatos de la Encuesta Nacional de Ingresos y Gastos de los Hogares (ENIGH) publicados por el Instituto Nacional de Estadística y Geografía (INEGI).

Códigos JEL: J01, J21, J23, J24, R40

Palabras clave: Economía laboral; Fuerza de trabajo y empleo; Tamaño y estructura; Demanda laboral; Elección ocupacional; Economía del transporte.

\footnotetext{
*Autor para correspondencia.

Correo electrónico: cruzgonzalezg@hotmail.com (G. Cruz González)

La revisión por pares es responsabilidad de la Universidad Nacional Autónoma de México.
} 


\begin{abstract}
In the present study the dynamics and structure of labor transport sector in Mexico is analyzed, specifically the "Drivers transport and mobile machinery" as well as the impact of economic growth on labor demand by categories of workers analyzed this sector according to the Classification of Occupations Unique of Mexican labor sector. The methodology presented is used by Kato (2004) following Sydsaeter and Hammond (1995) to determine the product elasticity of employment for the period 1996-2012, using microdata from National Survey of Income and Expenditure published by the National Institute of Statistics and Geography.
\end{abstract}

JEL codes: J01, J21, J23, J24, R40

Keywords: Labor Economy; Labor force and employment; Size and structure; Labor demand; Occupational choice; Transportation economy.

\title{
Introducción
}

El Gobierno de la República en su programa Sectorial de Comunicaciones y Transportes del Plan Nacional de Desarrollo 2013-2018, definió 3 estrategias transversales para llevar al país a su máximo potencial: 1) democratizar la productividad ${ }^{2}, 2$ ) gobierno cercano y moderno ${ }^{3}$ y 3) perspectiva de género ${ }^{4}$; el presente análisis se va a centrar en la primera estrategia, específicamente en el objetivo 1: "Fortalecer el buen funcionamiento de los mercados laborales para impulsar la creación de empleos formales bien remunerados" 5 particularmente del sector transporte. De tal forma, el propósito de este trabajo es analizar la dinámica y la estructura del sector laboral del transporte en México, así como el impacto del crecimiento económico sobre la demanda de trabajo por categorías de los trabajadores en el grupo: "Conductores de transporte y de maquinaria móvil" de acuerdo a la Clasificación Única de Ocupaciones (CUO) del sector laboral mexicano por entidad federativa. Además de identificar cuál de los diversos grupos unitarios que comprenden este grupo son los que tienen mayor respuesta al crecimiento del Producto Interno Bruto (PIB), de acuerdo a los resultados obtenidos en el cálculo de las elasticidades.

La dinámica del mercado laboral está ligada a múltiples factores; los diferentes niveles de especialización de la mano de trabajo, la globalización y la inclusión financiera puede influir de manera determinante en la demanda de mano de obra. Autores como Bauchet, Marshall, Starita, Thomas y Yalouris, 2011; Cull, R., T. Ehrbeck y N. Holle (2014), coinciden en que dichos fenómenos económicos tienen un efecto positivo en la dinámica del mercado laboral; sobre el autoempleo, la emancipación y el bienestar de los trabajadores. Según una

\footnotetext{
${ }^{2}$ La estrategia transversal "Democratizar la productividad", coordinará las acciones de gobierno encaminadas a llevar a cabo políticas públicas que eliminen los obstáculos que limitan el potencial productivo de los ciudadanos y las empresas; incentivar entre todos los actores de la actividad económica el uso eficiente de los recursos productivos; analizar de manera integral la política de ingresos y gastos públicos para que las estrategias y programas del gobierno induzcan la formalidad. Plan Nacional de Desarrollo 2013-2018. Programa Sectorial de Comunicaciones y Transportes.

${ }^{3}$ La estrategia transversal "Gobierno cercano y moderno", se establecerán mediante bases de colaboración suscritas entre la coordinadora de sector, la Secretaría de Hacienda y Crédito Público y la Secretaría de la Función Pública. Ibídem

${ }^{4}$ La estrategia transversal "Perspectiva de género", incorpora la igualdad de género en las políticas, programas, proyectos e instrumentos compensatorios de la Secretaría de Comunicaciones y Transportes, en especial en el Programa de Empleo y Empleo Temporal. Un país bien comunicado contribuye a evitar la discriminación. Ibídem.

${ }^{5}$ Ibídem.
} 
investigación reciente Fareed, F. et al. (2017), el acceso a los servicios financieros en México puede abrir oportunidades económicas para las mujeres, especialmente como emprendedoras. Estos resultados pueden resultar controvertibles, autores como González, G. (2004), señala que en México, las condiciones en que se desarrolla el mercado laboral en el contexto de la globalización económica, existen graves problemas para el crecimiento del producto y la generación de los empleos que demanda la población trabajadora.

Autores como Burgos, B. y López, K. (2010) y Samaniego, N., (2013), realizan por separado un estudio sobre el mercado laboral de profesionistas, buscando analizar la dinámica del mercado laboral por actividad profesional. Los autores elaboran un indicador de pertinencia para dicho mercado laboral. Los resultados, en general coinciden en que una proporción importante de profesionistas trabaja en áreas que no corresponde a su especialidad de carrera y muchas veces, en condiciones inadecuadas. Los mismos autores, también coinciden en que existen sesgos salariales en diferentes áreas profesionales.

En lo referente a literatura que se refiera a la dinámica laboral del sector transporte, es poco copiosa, sin embargo una revisión de la misma, nos permite aproximarnos de alguna manera al campo del análisis empírico de estudios sobre dicho sector. Trabajos como el de Islas, V., (1992, p.13), proporciona elementos que permiten definir el sector transporte como el conjunto de instituciones, personas e insumos que participan de manera directa o indirecta en la realización de un servicio que consiste en trasladar personas y/o bienes y servicios. La oferta de este servicio tiene su natural contrapartida en la demanda que de él presenten los demás sectores de la economía".

En el mismo artículo, Islas (1992, p. 13), menciona que de alguna manera, el éxito o fracaso de las actividades para el desarrollo de un país dependen en gran medida de los sistemas de transporte que coadyuvan en las actividades productivas de la economía. Ello hace del transporte objeto de interés, pues tiene un papel estratégico e insustituible dentro de la economía".

Es así que el presente análisis es de interés dentro de las acciones presentadas por el gobierno federal, pues el presente estudio busca dar un panorama de la actual situación laboral del sector transporte en México con la intención de impulsar la creación de empleos formales en donde éstos se demandan de acuerdo a las características que cada grupo unitario requiere.

Un documento publicado por el INEGI (2014) respecto al sector transporte en México en 2012, analiza el impacto del empleo dados los cambios en las unidades económicas de este sector, en esta publicación el INEGI señala que existen más de 25500 empresas del ramo de autotransporte de carga general, mientras que para la transportación marítima solo se contabilizaron 19 empresas; señala además que los ocupados en transporte vía terrestre representa poco más del $90 \%$ de los trabajadores del sector transporte. Así mismo señala la predominancia de los trabajadores hombres respecto de las mujeres, indicando que las mujeres son solo el $13.9 \%$ de personal ocupado en este sector, cifra muy similar a los cálculos propios (13.43\%). La publicación señala que en el año 2012 hubo una ligera pérdida de empleos (1\% aproximadamente) respecto al año anterior que en términos de unidades económicas representó a 51 empresas que dejaron de operar.

Por otra parte, respecto a la metodología, se utilizará la propuesta por Sydsaeter y Hammond (1995) en Kato (2004) para determinar la elasticidad producto del empleo, adicionalmente se identificarán las ocupaciones más dinámicas de este sector y que tienen una mayor respuesta al crecimiento del PIB. 
La investigación comprende un periodo de análisis de los años 1996 y 2012. Se hace uso de estadísticas generadas del manejo de información a nivel de microdatos de la Encuesta Nacional de Ingresos y Gastos de los Hogares (ENIGH) publicados por el INEGI. Mediante la elaboración de un análisis estadístico se calculará el efecto del crecimiento económico sobre el empleo del grupo "Conductores de transporte y de maquinaria móvil" en México para dicho periodo.

En estudios recientes el análisis de las elasticidades se ha empleado para estudiar la relación producto del empleo con la metodología propuesta por Sydsaeter y Hammond (1995), la cual retoma la CEPAL (2000) para un estudio de América Latina y el Caribe, misma metodología que también emplea Kato (2004) para su análisis de elasticidad producto del empleo en la industria manufacturera en México para el periodo 1987-2001 pero incorporando la variable de prestaciones sociales, las cuales el autor emplea para explicar los cambios en las magnitudes de las elasticidades; por su parte, Guerrero, C. (2007) cuantifica las elasticidades empleo a producto y salario en el Istmo Centroamericano haciendo un análisis de la evolución y perspectivas del mercado laboral en esa región.

Por otro lado, Ruiz, C. (2005), hace uso del cálculo de las elasticidades en un análisis sobre la integración de los mercados laborales en América del norte; Cruz, G. y Ríos, H. (2014), calculan las elasticidades producto del empleo para analizar la dinámica del sector laboral en México para diversas ocupaciones en el país; Ríos, J. y Carrillo, S. (2014), las utilizan en un análisis sobre el empleo en la manufactura de México, en el cual analizan el impacto del empleo ante cambios en el producto de las ramas de dicho sector a partir de la crisis del 2009; y finalmente Navarro (2009), quien presenta estimaciones sobre la dinámica del empleo en América Latina, calculando la elasticidad empleo-producto y la elasticidad empleo-salarios. Los trabajos que se han realizado para el análisis de la elasticidad producto del empleo en México han sido aplicados principalmente al sector manufacturero, sin embargo, la metodología que en ellos se utiliza se puede adaptar a cualquier sector de la economía que se desee analizar y sirven de guía para el análisis que aquí se va a presentar, en este caso al sector transporte en México.

El principal aporte de esta investigación, reside analizar de manera exhaustiva la dinámica y la estructura del sector laboral del transporte en México, lo cual permite determinar, el impacto del crecimiento económico sobre la demanda de trabajo por categorías de los trabajadores de dicho sector, de acuerdo a la Clasificación Única de Ocupaciones del sector laboral mexicano. Por otra parte, el trabajo muestra con argumentos empíricos que el crecimiento del producto afecta de manera desigual tanto a nivel nacional como por entidad federativa a cada uno de los grupos unitarios de los trabajadores del grupo 83.

El trabajo está integrado de la siguiente forma: el primer apartado contiene una exposición general de lo que es la CUO y los criterios para realizar la conformación del clasificador, así como una presentación de las actividades que realizan los trabajadores del grupo "Conductores de transporte y maquinaria móvil" proporcionada por el INEGI en la Encuesta Nacional de Ocupación y Empleo (ENOE). En el segundo apartado, se presenta un análisis de las estadísticas generadas a partir de microdatos de la ENIGH 1996 y 2012 de los "Conductores de transporte y maquinaria móvil" como son participación por género, grado de escolaridad, nivel de ingresos y prestaciones. En el tercer apartado se presenta por una parte, la revisión de la evidencia empírica contrastando los resultados obtenidos por diversos autores y los obtenidos en el presente análisis; y por otra parte, los resultados obtenidos del cálculo de las elasticidades por grupos unitarios de los "Conductores de transporte y maquinaria móvil" a nivel nacional y por entidad federativa. Finalmente, se presentan las conclusiones. 


\section{Descripción del grupo "Conductores de transporte y de maquinaria móvil"}

El análisis de los ocupados en el grupo "Conductores de transporte y de maquinaria móvil" será examinado a través de la Clasificación Única de Ocupaciones (CUO), el cual es un clasificador de ocupaciones que conforma los grupos principalmente en los conceptos de: 'empleo', 'ocupación', y 'competencia'6.

De acuerdo al INEGI (2009), la "CUO es un ordenamiento de ocupaciones principales en función de la división técnica del trabajo, también considera las situaciones derivadas de la problemática del empleo, como lo es el autoempleo. En esta clasificación se define la ocupación como el conjunto de funciones, obligaciones, actividades o tareas que desempeña un individuo en su empleo, oficio o puesto, independientemente de la actividad económica que se realice en el lugar donde éste trabaje y de las relaciones que establezca con los demás ocupados. Los criterios básicos bajo los cuales se realiza la conformación del clasificador, son los siguientes: i) la división técnica del trabajo, ii) el nivel de calificación de las ocupaciones y tareas realizadas, y iii) la utilización de instrumentos, herramientas y/o materias primas similares para el desempeño de las funciones de cada ocupación”.

De acuerdo a la $\mathrm{ENOE}^{7}$ (INEGI, 2009), "los trabajadores clasificados en este grupo (Conductores de transporte y de maquinaria móvil) controlan, atienden y operan maquinaria móvil durante el proceso de producción agropecuario, industrial, de la construcción, así como para la carga y descarga portuaria y movimiento de mercancías y comercios. Por ejemplo: los conductores de cosechadora, tractor, excavadora, asfaltadora, montacargas, etcétera. Este grupo comprende también a los conductores de trenes, metro y tren ligero, que transportan pasajeros o mercancías.

Incluye a los conductores de autobuses, camiones, automóviles. Taxis, trolebús, trailers, etcétera, para transportar pasajeros o mercancías. Se agrupa también a los conductores de naves aéreas (avión, avioneta, helicóptero) y de transportes marítimos (barcos, lanchas, botes) que transportan pasajeros o mercancías. También se incluyen a los conductores de vehículos de transporte de tracción humana (triciclos, carretas) y animales de carga (bueyes, mulas, caballos, burros). Se consideran en este grupo a los ayudantes de conductor, cuando realicen tareas auxiliares para el control y operación de los equipos de transporte. Se excluye de este grupo principal a los conductores de vehículos que promocionan, venden, entregan y cobran el producto o mercancía en establecimientos comerciales o en el domicilio de los clientes, ya que estos trabajadores se clasifican en el grupo unitario despachadores y dependientes de comercio".

La información que se presenta en la Tabla 1 se basa en los criterios utilizados en la CUO, el grupo 83 está formado por 6 subgrupos (tres dígitos) y 14 grupos unitarios (4 dígitos). Adicionalmente a este grupo, para efectos de este trabajo se están considerando tres grupos unitarios más, pues éstos se encontraban dentro de este grupo en la anterior clasificación los cuales se considera importante no dejarlos fuera en este análisis. Los grupos unitarios son los siguientes: 6311 Operadores de maquinaria agropecuaria y forestal, 9311 Ayudantes de conductor de transporte terrestre con motor, y 9321 Conductores de vehículos de transporte en bicicleta

\footnotetext{
${ }^{6}$ En 2008, en el marco de la reunión del Comité Sectorial de Estadísticas del Trabajo y Previsión Social, se acordó elaborarla con el objetivo de generar información que refleje las estructura ocupacional del país y comparable con otros sistemas de clasificación internacional. INEGI, Clasificación del Censo de Población y Vivienda 2010 , Julio de 2011.

${ }^{7}$ Instituto Nacional de Estadística y Geografía, ENOE-CMO, 2009, México.
} 
Tabla 1

Grupo 83: Conductores de transporte y de maquinaria móvil

830 Supervisores de conductores de transporte y de maquinaria móvil

8301 Supervisores de conductores de transporte y de maquinaria móvil

$831 \quad$ Conductores de transporte aéreo

$8311 \quad$ Pilotos de aviación

832 Conductores de transporte marítimo

$8321 \quad$ Capitanes y conductores de transporte marítimo

8322 Oficiales y marineros de cubierta y prácticos

8323 Oficiales maquinistas de transporte marítimo

8324 Conductores de pequeñas embarcaciones (lanchas, botes, trajineras, etc.)

833 Conductores de transporte en vías férreas

$8331 \quad$ Conductores de transporte en vías férreas (tren, metro, tren ligero)

834 Conductores de transporte terrestre con motor

8341 Conductores de camiones, camionetas, taxis y automóviles de carga

8342 Conductores de autobuses, camiones, camionetas, taxis y automóviles de pasajeros

$8343 \quad$ Choferes en casas particulares

$8344 \quad$ Conductor de motocicleta

8349 Otros conductores de transporte terrestre con motor no clasificados anteriormente

835 Conductores de maquinaria móvil

8351 Conductores de maquinaria móvil para la construcción y minería

8352

Conductores de maquinaria móvil para el movimiento de mercancías en fábricas, puertos, comercio, etc.

Fuente: Elaboración propia con base en Clasificación de Censo de Población y Vivienda 2010. INEGI, 2011.

\section{Análisis de los conductores de transporte y maquinaria móvil}

En este apartado se generaron estadísticas a partir de microdatos de la ENIGH 1996 y 2012. Para el año 1996 se hace una reclasificación de las ocupaciones de México, ya que hasta el 2008 se utilizaba la Clasificación Mexicana de Ocupaciones (CMO) la cual constaba de 19 grupos principales; a partir del 15 de julio de 2011 el INEGI publica una nueva clasificación llamada Clasificación Única de Ocupaciones la cual consta de 53 grupos principales ${ }^{8}$. Para los años 1996 y 2012, se dispone de información a nivel de microdatos de la ENIGH los cuales

\footnotetext{
${ }^{8}$ En esta reclasificación de las ocupaciones se hizo una homogeneización de las dos bases de datos, pues en la publicación de la ENIGH-2010, se cambia completamente la clasificación de ocupaciones, no solo en el nombre de dicha clasificación, pues hasta el 2008 se seguía llamando Clasificación Mexicana de Ocupaciones, y para el 2010 se publica en la ENIGH la nueva clasificación llamada Clasificación Única de Ocupaciones; además la clasificación de las ocupaciones cambia totalmente, por ejemplo: el grupo principal 11 en el año 1996 pertenecía a los Profesionistas; para el año 2010, el grupo 11 pertenece a los Funcionarios y altas autoridades del sector público, privado y social. Dentro de este grupo principal 11 existen subgrupos, por ejemplo en 1996 el subgrupo 111 pertenecía a los físicos, astrónomos, matemáticos, estadísticos y actuarios; para 2010 el subgrupo 111 pertenece a los funcionarios, legisladores y autoridades gubernamentales. Es por ello que fue necesario hacer una reclasificación de las ocupaciones de México para poder hacer el análisis comparativo entre los dos periodos de estudio del sector laboral mexicano.
} 
son ponderados mediante la variable de frecuencia factor de expansión para representar al total de los trabajadores del país. Se hace un análisis comparativo de los grupos unitarios de los trabajadores del grupo 83 para los años 1996 y 2012, de acuerdo a la CUO, tomando algunas características como son: personal ocupado, sexo, nivel de escolaridad, nivel de ingresos en salarios mínimos y prestaciones.

El grupo 83 está dentro de los grupos de ocupaciones más representativas de México, pues éste representa el 5.3\% para el año 1996 y el $4.7 \%$ para el 2012. En un análisis previo del sector laboral mexicano, Cruz, G. (2013), habla que de los 53 grupos principales de ocupación solo 22 son los más representativos, pues representan aproximadamente el $80 \%$ de la población económicamente activa ocupada del país, y el grupo de "Conductores de transporte y maquinaria móvil" está dentro de este grupo de las 22 ocupaciones más representativas, ocupando el $6^{\circ}$ lugar en orden de personas ocupadas. Por su parte, Ruiz (2005) en su trabajo sobre la integración de los mercados laborales, menciona que la población dedicada a la "Transportación, comunicaciones y almacenaje" es del 6\% del empleo total para el año 2003.

La tabla 2 muestra la participación de la población ocupada en el grupo: "Conductores de transporte y de maquinaria móvil". Se observa que para el año 2012 dos fueron los grupos unitarios con más trabajadores dentro de este grupo: el $46.80 \%$ de los ocupados fueron los conductores de autobuses, camiones, camionetas, taxis y automóviles de pasajeros; y el 35.90\% de los ocupados fueron conductores de camiones, camionetas y automóviles de carga. Estos dos grupos unitarios representan aproximadamente el $82 \%$ de los ocupados en este grupo.

Tabla 2

Participación de la población ocupada (\%). Grupo 83, 1996 y 2012

Grupo Unitario

Población Ocupada (\%)

Pilotos de aviación (8311)

1996

2012

Pilotos de aviación (8311)

1.01

0.07

Capitanes y conductores de transporte marítimo (8321)

0.39

0.08

Oficiales y marineros de cubierta y prácticos (8322)

0.03

0.03

Conductores de pequeñas embarcaciones (lanchas, botes, trajineras, etcétera) (8324)

0.24

0.16

Conductores de transporte en vías férreas (tren, metro y tren ligero) (8331)

0.15

0.04

Conductores de camiones, camionetas y automóviles de carga (8341)

36.66

Conductores de autobuses, camiones, camionetas, taxis y automóviles de pasajeros (8342)

Choferes en casas particulares (8343)

Otros conductores de transporte terrestre con motor, no clasificados anteriormente (8349)

Conductores de maquinaria móvil para la construcción y minería (8351)

Conductores de maquinaria móvil para el movimiento de mercancías en fábricas, puertos, comercio, etc. (8352) 
En la tabla se observa que los conductores de transporte en vías férreas, los conductores de transporte aéreo y los conductores de transporte marítimo son los que menor porcentaje de ocupados tienen en ambos periodos de tiempo, incluso en los cinco grupos unitarios de esos dos grupos principales (831 y 832) la población ocupada disminuyó excepto para los ocupados en el grupo 8322: Oficiales y marineros de cubierta y prácticos.

Respecto a la participación por género de la población ocupada en este sector, para el año de 1996 el $99.65 \%$ son hombres y el $0.35 \%$ son mujeres. La figura 1 muestra de manera detallada el grado de participación por género y por grupo unitario; se observa que el grupo unitario 9311 (Ayudantes de conductor de transporte terrestre con motor) y el grupo unitario 8342 (Conductores de autobuses, camiones, camionetas, taxis y automóviles de pasajeros) fueron los únicos grupos que tuvieron participación de la mujer, ambos con una participación pequeña del 3.19 y $0.53 \%$ respectivamente.

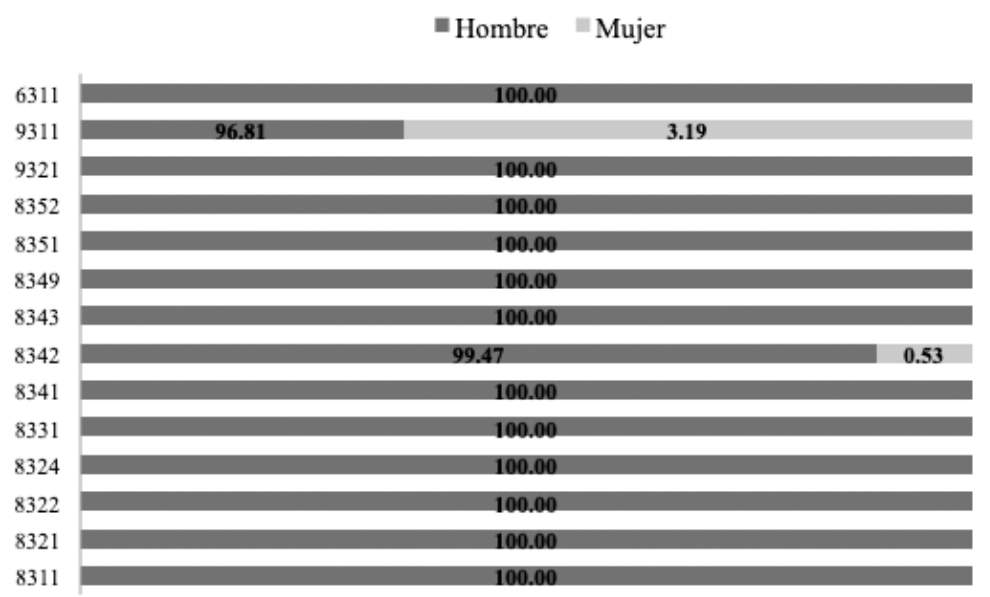

Figura 1. Participación por género de la población ocupada (\%). Grupo 83, 1996.

Fuente: Cálculos y elaboración propia con base en microdatos de la ENIGH 1996, INEGI.

El panorama cambia para el año de 2012, pues la participación de la mujer incrementó pasando del $0.35 \%$ registrado en 1996 a $13.43 \%$ de los trabajadores en este sector. La figura 2 muestra de manera detallada el grado de participación por género y por grupo unitario; se observa que el grupo unitario 9311 (Ayudantes de conductor de transporte terrestre con motor) incrementó la participación de la mujer de manera significativa pues su participación pasó de $3.19 \%$ a un $61.06 \%$ de los ocupados en este grupo, así mismo el grupo 8342 (Conductores de autobuses, camiones, camionetas, taxis y automóviles de pasajeros) pasó de 0.53 a $16.47 \%$ de participación de la mujer. Además de estos dos grupos unitarios, fueron seis más los que registraron participación de la mujer: el grupo 6311 (Operadores de maquinaria agropecuaria y forestal) con el 8.38\%; el grupo 9321 (Conductores de vehículos de transporte en bicicleta) con el 16.99\%; el grupo 8352 (Conductores de maquinaria móvil para el movimiento de mercancías en fábricas, puertos, comercio, etc.) con el 6.32\%; el grupo 8351 (Conductores de maquinaria móvil para la construcción y minería) con el 4.38\%; el grupo 8341 (Conductores de camiones 
y automóviles de carga) con el 9.90\%; y finalmente el grupo 8321 (Capitanes y conductores de transporte marítimo) con el $22.23 \%$.

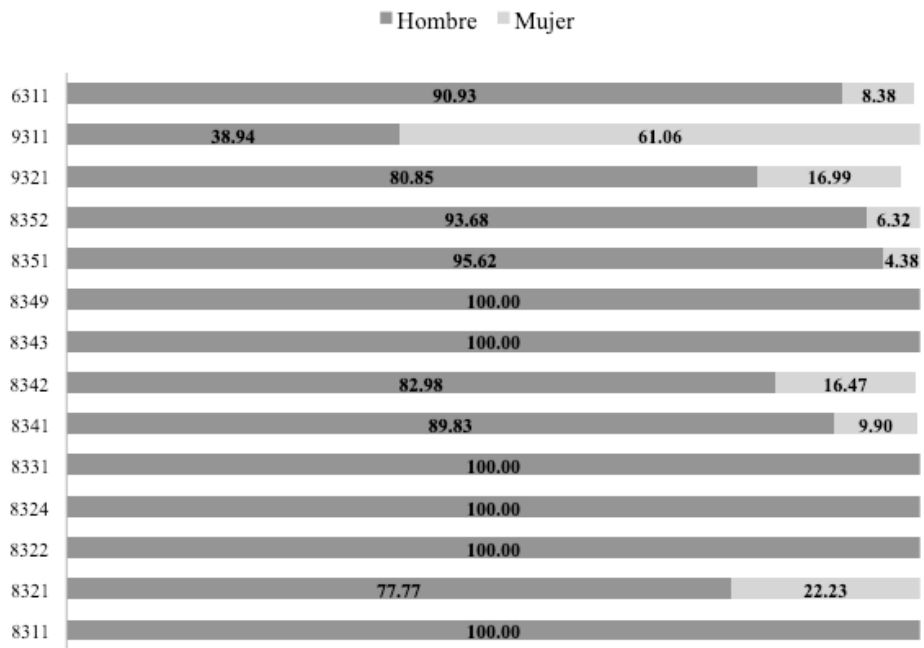

Figura 2. Participación por género de la población ocupada (\%). Grupo 83, 2012.

Fuente: Cálculos y elaboración propia con base en microdatos de la ENIGH 2012, INEGI.

Respecto a los niveles de instrucción formal para los trabajadores de este grupo, la figura 3 muestra el grado de escolaridad para el año 1996. Se observa que el nivel de escolaridad que predomina en los trabajadores de este grupo es el de primaria, seguido por el nivel de secundaria; cuatro grupos presentan estudios de nivel superior: el grupo 8311 (Pilotos de aviación) con 48.93\%, el grupo 8352 (Conductores de maquinaria móvil para el movimiento de mercancías en fábricas, puertos, comercio, etc.) con 7.14\%, el grupo 8341 (Conductores de camiones y automóviles de carga) con $0.82 \%$; y el grupo 8342 (Conductores de autobuses, camiones, camionetas, taxis y automóviles de pasajeros) con $0.38 \%$.

Para el año 2012, los niveles de instrucción formal para los trabajadores de este grupo mostraron cambios, la figura 4 muestra que el nivel de escolaridad que predomina es el de secundaria, seguido por el nivel medio superior; en este periodo se suman a los cuatro grupos con estudios de nivel superior tres más: el grupo 6311 (Operadores de maquinaria agropecuaria y forestal) con 1.58\%, el grupo 9311 (Ayudantes de conductor de transporte terrestre con motor) con $1.90 \%$, y el grupo 8351 (Conductores de maquinaria móvil para la construcción y minería) con $1.93 \%$. 


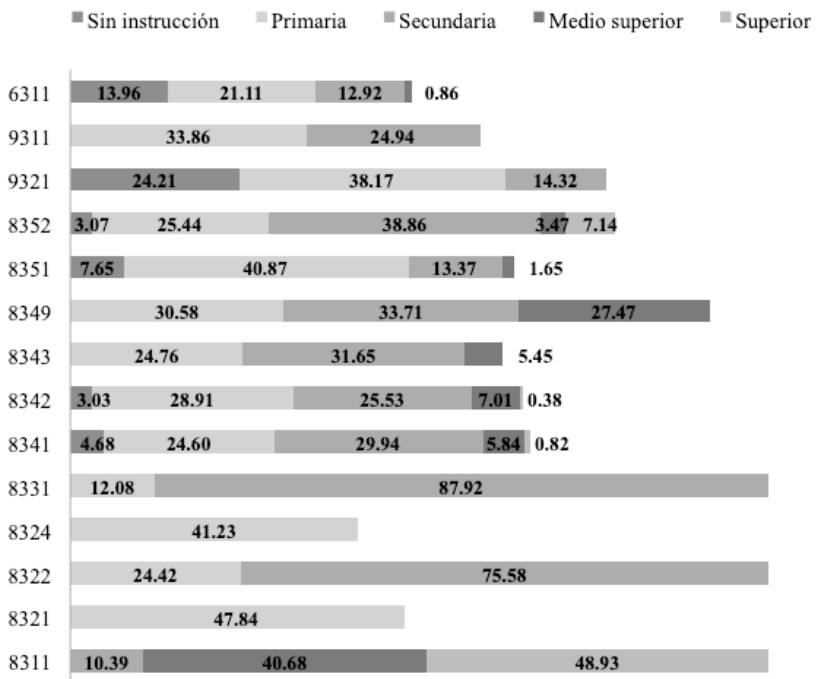

Figura 3. Grado de escolaridad de la población ocupada (\%). Grupo 83, 1996.

Fuente: Cálculos y elaboración propia con base en microdatos de la ENIGH 1996, INEGI.

"Sin instrucción "Primaria "Secundaria " Medio superior " Superior

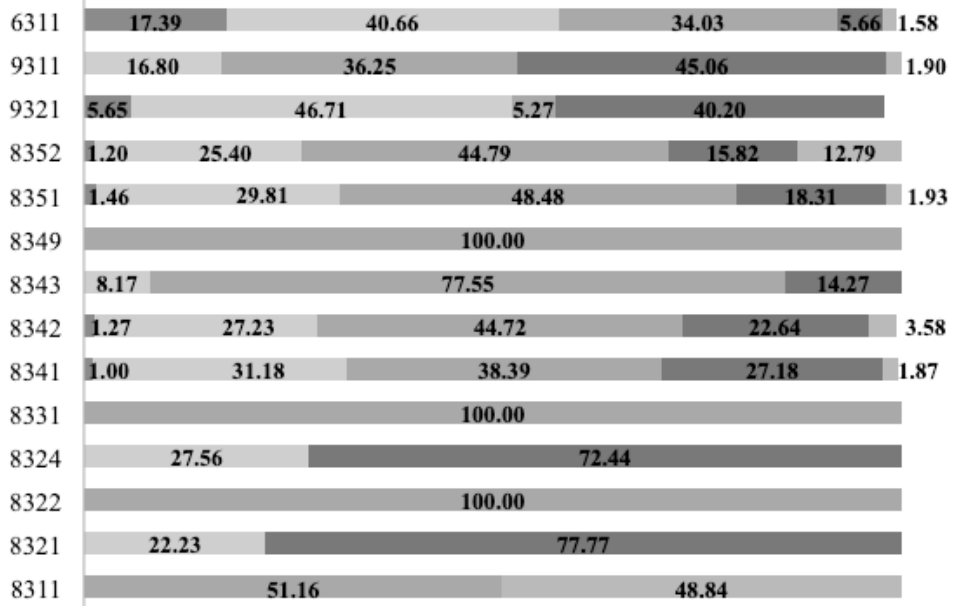

Figura 4. Grado de escolaridad de la población ocupada (\%). Grupo 83, 2012.

Fuente: Cálculos y elaboración propia con base en microdatos de la ENIGH 2012, INEGI. 
Respecto a las condiciones laborales, éstas fueron medidas respecto a si tenían o no prestaciones provenientes de su trabajo, la figura 5 muestra que un porcentaje considerable de los trabajadores de este grupo no tiene prestaciones. Para el año 1996 solo tres grupos principales tenían el 100\% de sus trabajadores prestaciones: el grupo 8311 (Pilotos de aviación), el grupo 8322 (Oficiales y marineros de cubierta y prácticos) y el grupo 8331 (Conductores de transporte en vías férreas). El grupo más precario fue el grupo 8324 (Conductores de pequeñas embarcaciones) pues el 71.75\% de sus trabajadores no tenían prestaciones. Para el año 2012 la situación no cambia mucho pues si bien hubo dos grupos más que tuvieron mejores condiciones por tener prestaciones: los grupos 8321 (Capitanes y conductores de transporte marítimo) y 8349 (Otros conductores de transporte terrestre con motor no clasificados anteriormente); el grupo 8311 tuvo un retroceso pues de contar anteriormente con el 100\% de sus trabajadores con prestaciones para el año 2012 solo el $48.84 \%$ se sus trabajadores tenían prestaciones.

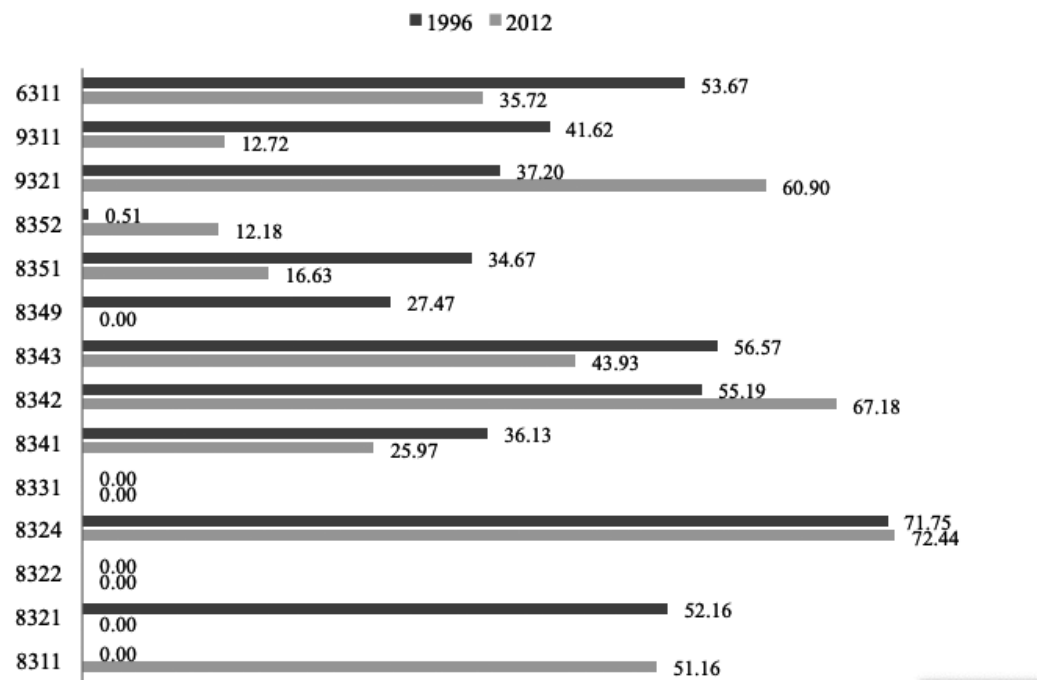

Figura 5. Población ocupada que no cuenta con prestaciones (\%). Grupo 83, 1996- 2012

Fuente: Cálculos y elaboración propia con base en microdatos de la ENIGH 1996- 2012, INEGI

Finalmente, la figura 6 muestra el nivel de ingresos de los trabajadores en 2012, clasificado en 6 categorías de Salario Mínimo General Promedio (SMGP), se observa que la mayoría de los trabajadores obtiene de 0 a 1 SMGP; solo tres grupos unitarios obtienen más de 10 SMGP: 6311 (Operadores de maquinaria agropecuaria y forestal) con el 3.81\%; y los grupos 8322 (Oficiales y marineros de cubierta y prácticos) y 8311 (Pilotos de aviación) con el 100\%. 


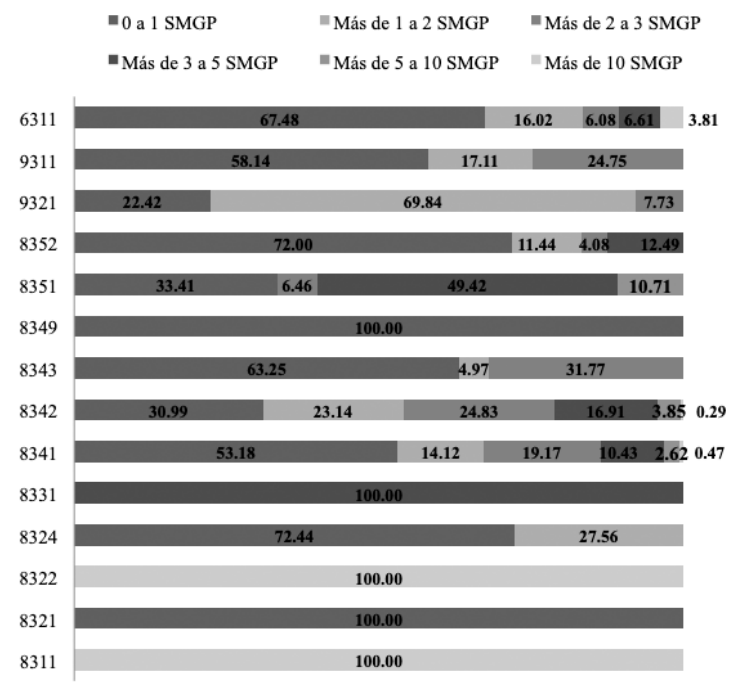

Figura 6. Nivel de ingresos SMGP* (\%). Grupo 83, 2012.

*Clasificación con base al Salario Mínimo General Promedio de los Estados Unidos Mexicanos, Secretaría del Trabajo y Previsión Social-Comisión Nacional de los Salarios Mínimos. (60.50 pesos diarios al 26 de noviembre de 2012).

Fuente: Cálculos y elaboración propia con base en microdatos de la ENIGH 2012, INEGI

\section{Evaluación de las elasticidades de los Conductores de transporte y de maquinaria móvil}

\section{Revisión de la evidencia empírica}

El objetivo principal de este trabajo es analizar el impacto del crecimiento económico sobre la demanda de trabajo por grupos unitarios de los ocupados en el grupo "Conductores de transporte y de maquinaria móvil" por categorías de la CUO, identificando así cuál de las diversas ocupaciones que están dentro de este grupo son las que tienen mayor respuesta para el país de acuerdo a los resultados obtenidos en el cálculo de elasticidades. Estudios muestran la evidencia empírica del análisis de la elasticidad producto del empleo aplicado principalmente al sector manufacturero en México en diferentes periodos de tiempo, sin embargo la metodología que en ellos se aplica puede ser empleada en cualquier sector de la economía; estos estudios sirven de guía para el análisis que aquí se presenta.

Ruiz (2005) en su trabajo sobre la integración de los mercados laborales, presenta un análisis de nichos de empleo a través de un análisis de la elasticidad producto del empleo, en el cual obtiene que a nivel nacional para el periodo de 1994-2000, el crecimiento del país es intensivo, no así para el periodo de 1987-1993; la comparación que hace el autor a nivel nacional, lo hace con respecto a Estados Unidos y Canadá, por lo que al final observa que México presenta una pérdida en ambos periodos de tiempo. La estimación de elasticidad producto del empleo que obtuvo a nivel nacional para el periodo 1987-1993 en el sector de "Transporte, comunicaciones y almacenaje" fue de 0.81 y para el periodo 1994-2000 fue de 0.58. Por su parte, Cruz y Ríos (2014), obtienen de la estimación de la elasticidad producto del empleo de los "Conductores 
de transporte y maquinaria móvil" para el periodo 1996-2010 un valor de 0.75. En el caso particular de esta investigación, el cálculo se hace de manera más detallada para el grupo "Conductores de transporte y de maquinaria móvil", obteniendo el cálculo de las elasticidades a nivel de grupo unitario tanto del total nacional como por entidad federativa, lo cual enriquece el análisis para aquellos que deseen una consulta más detallada sobre dicho sector.

Guerrero (2007), hace un análisis de elasticidades aplicada no solo al empleo sino que al mismo tiempo la aplica al salario, es decir, hace cálculos de elasticidad empleo a producto y salario en la región centroamericana para el periodo 1980-2004, en donde impone como restricción que la demanda del empleo dependa solamente del nivel de actividad económica. Una de los principales resultados a que el autor llega con este análisis es que: "los valores de las elasticidades empleo formal a producto son, reducidos por sí mismos, y bastante menores que los del empleo total. Por tanto, los resultados representan evidencia favorable a la hipótesis, que afirma la existencia de un sesgo en materia laboral de modelo de desarrollo vigente en esa región, en el sentido de su limitada capacidad para generar empleos de calidad" (Guerrero, 2007 , p. 19). Dicha hipótesis coincide con los resultados obtenidos en el presente análisis al compararlos con los valores negativos de las elasticidades, los cuales se ven reflejados en la disminución de la población ocupada en ciertos grupos unitarios del sector transporte.

Ríos y Carrillo (2009), en su análisis sobre empleo calificado y no calificado, presentan los valores de la elasticidad empleo-producto y empleo-salario del sector manufacturero en México a partir de la crisis del 2009. El autor refiere que el coeficiente de la elasticidad empleo-producto de la demanda de trabajo en el sector manufacturero va a depender del tipo de subsector, de la empresa y de los empleos generados para que éste cumpla con la teoría económica de ser positivo. Una medida de política económica que sugiere el autor para la conservación de los empleos, es la de preservar el empleo aun siendo no calificado en el sector manufacturero, esto para no impactar más de manera negativa ante los impactos económicos de la crisis en ese periodo de tiempo. Hace mención de los programas que implementó el gobierno para la preservación del empleo, cuyas medidas trataban de conservar el mayor número de empleos a cambio de un salario más bajo pero con la promesa de evitar la pérdida de éstos, así como apoyo a las empresas por medio de un subsidio a cambio de mantener el empleo de sus trabajadores. Sin embargo dado el resultado de la elasticidad empleo-producto, el autor menciona que dicho apoyo no fue suficiente para algunos subsectores de la manufactura como fue: fabricación de prendas de vestir y fabricación de muebles. Otra conclusión importante a la que llega el autor es que "los subsectores de alta tecnología no son los únicos con una mayor elasticidad producto-empleo y que, por lo mismo, ante una crisis económica resultarían más afectados en la reducción consecuente de empleos" (Ríos y Carrillo, 2009, p. 710). Esta conclusión a la que llegan los autores coincide con el valor del cálculo de elasticidad obtenido en el grupo 8311 (Pilotos de aviación), pues la tecnología que en ese grupo se utiliza es mayor, y por lo tanto al haber una crisis económica los empleos se ven más afectados, tal fue el caso ante la crisis de 2010 que presentó este grupo, y cuyo resultado se vio reflejado en el valor negativo de elasticidad que se obtuvo en los cálculos que aquí se presentan. No así para el caso del grupo 8341 (Conductores de camiones, camionetas y automóviles de carga) que aunque tuvo una disminución en el personal ocupado, este resultado no impacto de manera negativa al valor obtenido de la elasticidad, lo cual pudiera atribuirse entre otras causas, a que los procesos que en este grupo se emplea no son de alta tecnología. 
Navarro (2009), hace un análisis de la dinámica del empleo para 15 países de América Latina, calculando la elasticidad empleo-salario, en la cual "si ésta cae durante las recesiones, las políticas destinadas a reducir el costo laboral tendrían una limitada efectividad en una recesión como la actual (2009)" (Navarro, 2009, p. 26). Sus cálculos dan como resultado "elasticidades empleo-producto y empleo-salarios de signos consistentes con la teoría y evidencia empírica internacional". Una conclusión importante, es que "en los resultados se predice una mayor elasticidad empleo-producto y una menor elasticidad empleo-salario durante las fases de desaceleración y contracción de producto. Esto sugiere que políticas destinadas a reducir costos laborales tendrían un impacto limitado para disminuir el desempleo durante las recesiones. En cambio, y de acuerdo con los resultados, las políticas destinadas a estimular la demanda agregada fortalecerían su impacto positivo en el empleo en tiempo de crisis" (Navarro, 2009, p. 38).

Finalmente, revisando la metodología presentada por Kato (2004) siguiendo a Sydsaeter y Hammond (1995) que consiste en determinar la elasticidad producto del empleo la cual calcula la elasticidad dividiendo la tasa de crecimiento del empleo entre la tasa de crecimiento del producto, esta estimación consiste básicamente en medir cuánto crece, en promedio, el empleo al aumentar la producción. Esta metodología es la que se va a emplear en el análisis que aquí se presenta para cuantificar el efecto del crecimiento económico sobre el empleo en México para el periodo 1996-2012 con la particularidad de aplicarlo al grupo: "Conductores de transporte y de maquinaria móvil” a nivel de microdatos y por entidad federativa de cada grupo unitario contenido en este grupo de trabajo.

Kato utiliza una variable adicional: prestaciones sociales, para explicar los cambios en las magnitudes de las elasticidades del sector manufacturero, proponiendo como hipótesis "que en México la creación de empleos ha tenido poco aumento, no sólo por las bajas tasas de crecimiento de la producción, sino también por el encarecimiento del insumo trabajo, al tener que cubrir sus prestaciones sociales" (Kato, 2004, p. 91-92). El autor llega a la conclusión de que la elasticidad producto del empleo tiende a ser mayor en el sector manufacturero que en total de la economía; además de que contrasta la hipótesis antes mencionada con evidencia empírica, la cual dio como resultado que "las prestaciones sociales se redujeron en promedio por trabajador y permiten una expansión del nivel de empleo en la economía" (Kato, 2007, p. 95), es decir, se sacrifican las prestaciones por la creación de empleos. En este caso, no se toma en cuenta esa variable, sin embargo, como se observó en la figura 5 hay un porcentaje considerable de trabajadores que no cuenta con prestaciones laborales en ambos periodos de tiempo.

\section{Análisis de la elasticidad producto del empleo para los "Conductores de transporte y de maquinaria móvil"}

De acuerdo a Kato9 (2004), y entre otros autores, "se postula que el empleo es una función del nivel de producción como propone el esquema de mercado de trabajo. Por ello, la relación empleo-producto se expresaría de la siguiente manera:

$$
\mathrm{L}=\mathrm{f}(\mathrm{Q})
$$

\footnotetext{
${ }^{9}$ Kato, E. (2004). "Elasticidad producto del empleo en la industria manufacturera mexicana", Problemas del desarrollo, vol. 35, núm. 138, pp. 87-96, México
} 
Donde:

L es el nivel de empleo

$\mathrm{Q}$ es el nivel de producción, $\mathrm{f}^{\prime}>0$ y $\mathrm{f}^{\prime \prime}<0$, lo cual indica que el empleo crece conforme aumenta la producción, aunque a tasas decrecientes.

Ahora bien, para estimar la elasticidad producto del empleo, primero se definen las variaciones absolutas y relativas siguiendo a Sydsaeter y Hammond (1995). Formalmente se puede definir la variación absoluta del empleo así:

$$
\Delta \mathrm{L}=\mathrm{f}(\mathrm{Q}+\Delta \mathrm{Q})
$$

En la cual, el cambio en el empleo $\Delta \mathrm{L}$ es una función del cambio en la producción $\Delta \mathrm{Q}$ y de su nivel actual Q, previo al cambio. Otra medida de sensibilidad del cambio en el empleo, adicional a la variación absoluta, puede ser la pendiente de la función (1), ésta es $\Delta \mathrm{L} / \Delta \mathrm{Q}$.

Sin embargo, tanto la variación absoluta como la pendiente tienen un problema de comparación, dadas las unidades de medida, puesto que, si se quisiera comparar la industria moderna con la producción artesanal, se tendría una alta dispersión de los valores que se contrasta, debido a la naturaleza inherente de las actividades; así mismo, sería alto el diferencial de productividad e intensidad en el uso del capital.

Un procedimiento técnico que resuelve esta dificultad de comparación es la estandarización del cambio absoluto entre el nivel de las variables. A esto se le conoce como variación relativa o tasa de crecimiento. En el caso de la variable de empleo se expresa como:

$$
\frac{\Delta \mathrm{L}}{\mathrm{L}}=\frac{\mathrm{f}(\mathrm{Q}+\Delta \mathrm{Q})-\mathrm{f}(\mathrm{Q})}{\mathrm{Q}}
$$

De esta definición se construye en la ecuación (4) el concepto de elasticidad producto del empleo, el cual es el resultado de dividir el cambio relativo del empleo entre el cambio relativo de la producción:

$$
\text { Elasticidad producto del empleo }=\frac{\text { cambio relativo del empleo }}{\text { cambio relativo del producto }}
$$

De acuerdo a Sydsaeter y Hammond (1995), este cociente está libre de unidades de medida y sólo indica cómo responde el nivel de empleo a una variación del nivel de producción.

Así, un incremento de $10 \%$ en el empleo es la misma variación proporcional, independientemente si existen cien mil o un millón de empleos en la economía. Entonces, la variación para el empleo es:

$$
\frac{\frac{\Delta L}{L}}{\frac{\Delta Q}{Q}}=\frac{Q \Delta L}{L \Delta Q}=\frac{Q}{f(Q)} \cdot \frac{f(Q+\Delta Q)-f(Q)}{\Delta Q}
$$


Ahora sustituyendo $\frac{\Delta \mathrm{L}}{\mathrm{L}}$ de la ecuación (3) en la ecuación (4), se obtiene:

$$
\varepsilon_{\mathrm{LQ}}=\frac{\mathrm{Q}}{\mathrm{f}(\mathrm{Q})} \cdot \frac{\mathrm{df}(\mathrm{Q})}{\mathrm{dQ}}
$$

Entonces $\boldsymbol{\varepsilon}_{\mathrm{LQ}}$ se calcula como el resultado de la multiplicación de la productividad media del trabajador por la pendiente del empleo en relación con un cambio marginal de la producción; se determinan las elasticidades, como se sugiere en la ecuación (4), mediante la división de los cambios relativos" $"$.

En esta investigación se desarrolla un análisis para comprobar la hipótesis en la dirección propuesta por estos autores, calculando la elasticidad producto del empleo, con la particularidad como ya se ha mencionado anteriormente de llevar a cabo el análisis por grupos unitarios de los trabajadores ocupados en el grupo de "Conductores de transporte y de maquinaria móvil" de México y por entidad federativa a nivel de microdatos.

La figura 7 muestra el resultado del cálculo de las elasticidades para el periodo 1996-2012 de los "Conductores de transporte y de maquinaria móvil" a nivel nacional; se observa que de los 14 grupos, 5 presentaron elasticidades negativas. El grupo que presentó la elasticidad más alta fue el 9321 (Conductores de vehículos de transporte en bicicleta) con un valor de 2.248, esto pudiera deberse al incremento que hubo por la implementación de programas en este tipo de vehículos. Por otra parte, el grupo unitario con la elasticidad más baja fue el 8311 (Pilotos de aviación) con un valor de -4.343 , lo cual pudiera deberse a la crisis que se enfrentó en 2010 por parte de la aerolínea Mexicana de Aviación la cual suspendió operaciones para después declararse en quiebra. Otra de las conclusiones a las que llegan Ríos y Carrillo (2014) y que coincide con el resultado de la figura 7, es que las elasticidades positivas corresponden en buena medida al empleo no calificado, en este caso los grupos unitarios que presentaron elasticidades positivas en su mayoría tienen un grado de escolaridad de nivel secundaria. Nuevamente, el caso del grupo 8311, coincide con lo que Kato (2004) refiere sobre los incrementos en el desempleo, lo cual lo atribuye a una disminución del valor obtenido en la elasticidad producto del empleo, pues este grupo tuvo una valor en su elasticidad de -4.343 , pasando de 1.01 a $0.07 \%$ de su población ocupada en el periodo de 1996-2012, conclusión similar aunque se traten de sectores distintos de la economía mexicana, pues Kato se refiere a las elasticidad producto del empleo en la industria manufacturera y aquí se trata sobre el sector transporte en México.

A nivel estatal, los grupos que tuvieron total participación en las 32 entidades federativas fueron los trabajadores del grupo 8341: Conductores de camiones, camionetas, taxis y automóviles de carga; y los trabajadores del grupo 8342: Conductores de autobuses, camiones, camionetas, taxis y automóviles de pasajeros. El resto de los grupos unitarios no tuvo participación total en las 32 entidades federativas como se presenta en la Tabla 3, en los resultados obtenidos en el cálculo de las elasticidades para cada una de las entidades federativas.

\footnotetext{
${ }^{10}$ Kato, E., (2004). "Elasticidad producto del empleo en la industria manufacturera mexicana", Problemas del desarrollo, vol. 35, núm. 138, pp. 87-96, México.
} 


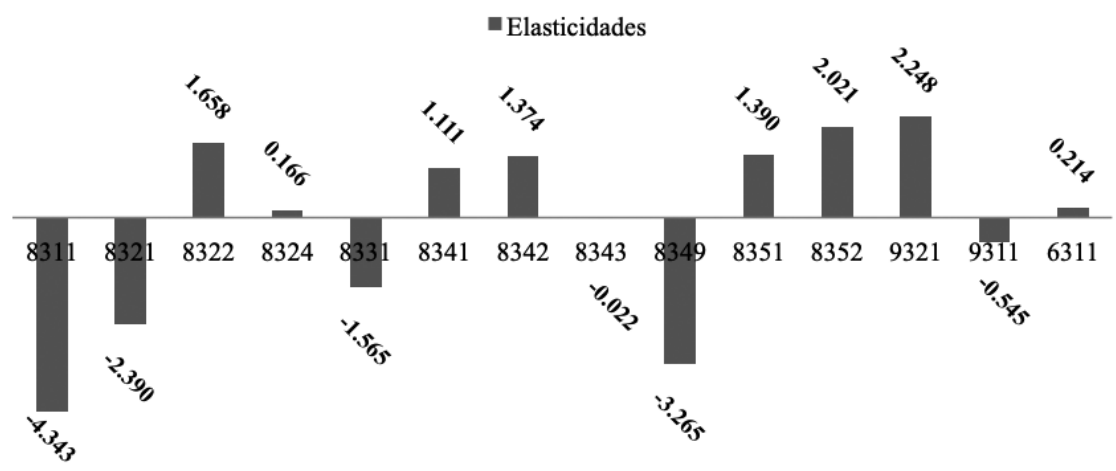

Figura 7. Elasticidades: Conductores de transporte y de maquinaria móvil, 1996- 2012

Fuente: Cálculos y elaboración propia con base en microdatos de la ENIGH 1996-2012. SCNM-PIB Anual, 1996-2012, Año base 2008. INEGI

Del análisis gráfico presentado por los resultados obtenidos de los cálculos de las elasticidades de los "Conductores de transporte y de maquinaria móvil" por entidad federativa, se pude concluir lo siguiente: El primero y que ya se había mencionado más arriba, dos son los grupos que tiene presencia en las 32 entidades federativas: el grupo unitario 8341 y el 8342 . Segundo, en la Tabla 3 se puede observar que no todos los grupos unitarios tienen presencia en las 32 entidades federativas, tal es el caso del grupo unitario 8331 el cual solo tuvo participación en Campeche y México con una elasticidad positiva y negativa respectivamente. Tercero, hubo entidades en los que las actividades registraron elasticidades con valores altos negativos por lo que su PEAO presentó valores muy bajos, y por el contrario hubo actividades en las que incrementaron la participación de su PEAO debido a las elasticidades positivas. Cuarto, las entidades federativas que tuvieron una mayor participación de la PEAO en este grupo principal fueron: México, Veracruz, Distrito Federal, Jalisco, Michoacán, Nuevo León y Puebla; por otra parte, las entidades federativas con menor participación de su PEAO en este grupo principal fueron Baja California Sur, Colima, Campeche, Tlaxcala, Aguascalientes y Zacatecas. Finalmente, respecto a las elasticidades se observa que tiene diferente comportamiento un mismo grupo unitario en cada una de las entidades federativas donde tiene participación de su PEAO, por ejemplo, para el grupo unitario 8351 el estado de Chihuahua tuvo una elasticidad negativa de -0.073 , no así para el estado de Hidalgo que presentó una elasticidad positiva de 0.487 . 
Tabla 3

Elasticidades por Entidad Federativa: Conductores de transporte y de maquinaria móvil, 1996-2012

\begin{tabular}{|c|c|c|c|c|c|c|c|c|c|c|c|c|c|c|}
\hline & 8311 & 8321 & 8322 & 8324 & 8331 & 8341 & 8342 & 8343 & 8349 & 8351 & 8352 & 9321 & 9311 & 6311 \\
\hline AGUASCALIENTES & 0.000 & 0.000 & 0.000 & 0.000 & 0.000 & 1.226 & 0.108 & -2.485 & 0.000 & -2.485 & 0.239 & 0.000 & 0.000 & -3.091 \\
\hline BAJA CALIFORNIA & 0.000 & 0.000 & 0.000 & 0.000 & 0.000 & 0.133 & -0.168 & 0.000 & 0.000 & 5.161 & 7.206 & 0.000 & 0.000 & -0.176 \\
\hline $\begin{array}{ll}\text { BAJA } & \text { CALIFORNIA } \\
\text { SUR } & \end{array}$ & 3.692 & 3.170 & 3.604 & -2.000 & 0.000 & 0.014 & -0.009 & 0.000 & 0.000 & -0.169 & -2.225 & 0.000 & 3.604 & 2.808 \\
\hline CAMPECHE & 2.406 & 0.000 & -1.357 & -1.221 & 0.405 & 0.189 & 0.286 & 0.470 & 0.000 & 0.502 & 0.385 & 0.119 & -1.395 & 2.857 \\
\hline COAHUILA & 0.000 & 0.000 & 0.000 & 0.000 & 0.000 & -0.175 & 0.083 & 0.000 & -2.649 & -3.113 & 0.752 & 0.000 & -0.137 & -2.923 \\
\hline COLIMA & 0.000 & 0.000 & 0.000 & 4.277 & 0.000 & 0.355 & 0.599 & 0.000 & 0.000 & -0.082 & -0.200 & 0.000 & 0.000 & -2.586 \\
\hline CHIAPAS & 0.000 & 0.000 & 0.000 & 0.000 & 0.000 & 0.067 & 0.879 & 5.514 & 0.000 & -3.167 & 0.000 & 0.000 & 0.000 & -2.932 \\
\hline CHIHUAHUA & 0.000 & 0.000 & 0.000 & 0.000 & 0.000 & 0.389 & 0.382 & 0.000 & 0.000 & -0.073 & 6.747 & 0.000 & 0.290 & -3.811 \\
\hline DISTRITO FEDERAL & -4.244 & 0.000 & 0.000 & 0.000 & 0.000 & 0.367 & -0.243 & 0.041 & -4.031 & 0.000 & -3.453 & 7.236 & -4.396 & 0.000 \\
\hline DURANGO & 0.000 & 0.000 & 0.000 & 0.000 & 0.000 & 0.297 & -0.009 & 0.000 & -3.003 & 0.000 & 0.184 & 4.970 & -3.186 & 6.128 \\
\hline GUANAJUATO & 0.000 & 0.000 & 0.000 & 0.000 & 0.000 & 0.175 & -0.004 & 0.000 & 0.000 & -2.903 & -2.456 & 0.000 & -2.971 & 6.412 \\
\hline GUERRERO & 0.000 & 0.000 & 0.000 & 5.741 & 0.000 & 0.211 & 0.937 & 0.000 & 0.000 & 0.000 & 0.000 & 5.187 & -3.535 & -3.374 \\
\hline HIDALGO & 0.000 & 0.000 & 0.000 & 0.000 & 0.000 & 0.317 & 0.211 & -2.444 & 0.000 & 0.487 & -2.477 & 0.000 & 0.577 & 0.393 \\
\hline JALISCO & 0.000 & 0.000 & 0.000 & 0.000 & 0.000 & 0.272 & 0.301 & -0.037 & 0.000 & 1.065 & 0.520 & 0.000 & 0.000 & 1.302 \\
\hline MÉXICO & -3.652 & 0.000 & 0.000 & 0.000 & -3.284 & 0.336 & 0.511 & 0.354 & -2.792 & 0.000 & 0.512 & 0.628 & -0.164 & -3.294 \\
\hline MICHOACÁN & 0.000 & 0.000 & 0.000 & 0.000 & 0.000 & 0.206 & 0.527 & 0.000 & 0.000 & 5.802 & 0.000 & 0.000 & 0.000 & 5.208 \\
\hline MORELOS & 0.000 & 0.000 & 0.000 & 0.000 & 0.000 & -0.003 & 0.161 & 4.889 & 5.102 & 5.971 & 0.000 & 0.000 & 0.000 & 0.000 \\
\hline NAYARIT & 0.000 & 0.000 & 0.000 & 0.000 & 0.000 & 0.581 & 0.332 & 0.000 & 0.000 & 4.329 & 0.587 & 0.000 & -0.393 & -0.447 \\
\hline NUEVO LEÓN & 0.000 & 0.000 & 0.000 & 0.000 & 0.000 & 0.264 & -0.113 & 0.000 & 0.000 & 5.008 & 0.668 & 0.000 & -2.682 & -2.741 \\
\hline OAXACA & 0.000 & 0.000 & 0.000 & -2.436 & 0.000 & -0.503 & 0.388 & -2.622 & 0.000 & 1.503 & 0.511 & -2.769 & 0.000 & -2.946 \\
\hline PUEBLA & 0.000 & 0.000 & 0.000 & 0.000 & 0.000 & 0.457 & 0.461 & -3.555 & 0.000 & 0.000 & -3.540 & 0.000 & 0.000 & 0.000 \\
\hline QUERÉTARO & 0.000 & 0.000 & 0.000 & 0.000 & 0.000 & 0.383 & -0.113 & -0.027 & 0.000 & 3.871 & 0.052 & 0.000 & 4.894 & 4.058 \\
\hline QUINATANA ROO & 0.000 & 4.217 & 0.000 & -2.534 & 0.000 & 0.716 & 0.268 & 0.000 & 0.000 & -0.444 & 0.000 & 0.000 & 4.134 & -2.518 \\
\hline SAN LUIS POTOSÍ & -2.829 & 0.000 & 0.000 & 0.000 & 0.000 & 0.166 & 0.167 & 0.000 & 0.000 & -2.832 & -3.149 & 0.000 & -2.813 & 4.579 \\
\hline SINALOA & 0.000 & 0.000 & 0.000 & 0.000 & 0.000 & 0.391 & -0.002 & 0.000 & 0.000 & -3.501 & 0.000 & 0.000 & 0.000 & -0.156 \\
\hline SONORA & 0.000 & 0.000 & 0.000 & 0.000 & 0.000 & 0.213 & 0.510 & 0.000 & 0.000 & 0.745 & 4.956 & 0.000 & 0.000 & -3.538 \\
\hline TABASCO & 0.000 & 0.000 & -1.284 & -1.319 & 0.000 & -0.060 & 0.681 & 0.000 & 0.000 & 0.006 & 0.000 & 0.000 & -1.567 & -0.002 \\
\hline TAMAULIPAS & 0.000 & -3.177 & 0.000 & -3.058 & 0.000 & 0.328 & 0.253 & -2.911 & 0.000 & 0.000 & 0.000 & 0.000 & 0.000 & -0.722 \\
\hline TLAXCALA & 0.000 & 0.000 & 0.000 & 0.000 & 0.000 & 0.288 & 0.390 & -2.540 & 0.000 & -2.488 & 0.847 & 0.000 & 0.000 & 0.000 \\
\hline VERACRUZ & 0.000 & -2.965 & 0.000 & 0.000 & 0.000 & 0.122 & 0.452 & 0.000 & 0.000 & 5.544 & 0.798 & 0.000 & 5.015 & 5.490 \\
\hline YUCATÁN & 0.000 & 0.000 & 0.000 & 0.000 & 0.000 & 0.471 & -0.136 & 0.000 & -2.722 & 4.167 & 0.000 & 0.044 & 4.525 & 4.167 \\
\hline ZACATECAS & 0.000 & 0.000 & 0.000 & 0.000 & 0.000 & 0.004 & 0.636 & 0.000 & 0.000 & 3.827 & 0.000 & 0.000 & 0.000 & -0.309 \\
\hline
\end{tabular}




\section{Conclusiones}

Retomado la idea central del objetivo del trabajo, se ha comprobado por una parte que el crecimiento del producto afecta de manera desigual tanto a nivel nacional como por entidad federativa a cada uno de los grupos unitarios de los trabajadores del grupo 83. Se identificó cuál de las diversas ocupaciones que existen en el grupo de "Conductores de transporte y de maquinaria móvil" son las que tienen mayor respuesta para el país de acuerdo a los resultados obtenidos en el cálculo de las elasticidades, ya que se obtuvieron elasticidades con valores positivos y elasticidades con valores negativos; las condiciones laborales de estos subgrupos son totalmente distintas pues los niveles de escolaridad y de ingreso salarial pudieran estar en los extremos.

Por otra parte, el fuerte de la PEAO del grupo 83, tiene más de uno y hasta tres salarios mínimos, son trabajadores con niveles de escolaridad baja (primaria y secundaria), además de la existencia de una mayor participación de hombres que de mujeres, situación que tal vez se deba a la propia naturaleza del sector y no a una discriminación de género. Cabe señalar que las condiciones del mercado laboral no son del todo favorables pues la mayoría de los empleos que se generan son con bajos salarios o sin prestaciones sociales. Como lo mencionó Guerrero (2007) en su análisis de empleo, se deben crear empleos suficientes y de calidad como principal estrategia de desarrollo, esta conclusión aplica no sólo a los seis países que él analiza, pues con el presente análisis observamos que el sector transporte de México requiere de la generación de empleos de calidad: con mejores salarios, prestaciones y capacitación al personal de acuerdo al grupo en el que desempeña su trabajo.

Hubo estados en los que las actividades registraron elasticidades con valores negativos por lo que la participación de su PEAO registró valores muy bajos; y por el contrario hubo actividades en las que se registraron elasticidades con valores positivos reflejándose en un incremento de la participación de su PEAO en 16 años. Las entidades federativas que tuvieron una mayor participación de la PEAO en este grupo principal fueron: México, Veracruz, Distrito Federal, Jalisco, Michoacán, Nuevo León y Puebla; por otra parte, las entidades federativas con menor participación de se PEAO fueron: Baja California Sur, Colima, Campeche, Tlaxcala, Aguascalientes y Zacatecas.

Respecto a la participación de los grupos unitarios, los que tuvieron más participación de su PEAO fueron: el grupo 8342: Conductores de autobuses, camiones, camionetas, taxis y automóviles de pasajeros, el grupo 8341: Conductores de camiones, camionetas, taxis y automóviles de carga, el grupo 8352: Conductores de maquinaria en movimiento de mercancías en fábricas, puertos, comercio, etcétera. Los grupos unitarios con baja participación de su PEAO fueron: el grupo 8322: Oficiales y marineros en cubierta y prácticos, el grupo 8331: Conductores de transporte en vías férreas, y el grupo 8321: Capitanes y conductores de transporte marítimo. Se observa que las elasticidades de un mismo grupo unitario tienen diferente comportamiento en cada una de las entidades federativas, presentando elasticidades positivas en un estado y negativas en otro. Las entidades federativas que se vieron favorecidas en el crecimiento de la PEAO empleada en este grupo principal "Conductores de transporte y de maquinaria móvil" dado un aumento en la producción y que es resultado principal del análisis del cálculo de las elasticidades fueron: Campeche, Jalisco, Michoacán, Morelos, Sonora. Veracruz y Yucatán, sólo por mencionar los estados en los que se registraron más elasticidades positivas que negativas. 
Es así como esta investigación da la pauta para generar insumos para la toma de decisiones en las políticas públicas, como puede ser la construcción de centros de capacitación a trabajadores para mejorar su calidad de vida, capacitándose en oficios acorde a la demanda laboral de este sector en particular y el fomento a emprendedores, específicamente para los "Conductores de transporte y de maquinaria móvil" enfocadas a las necesidades de cada entidad federativa. Por otra parte, en algunos grupos unitarios, el porcentaje de la población que no cuenta con prestaciones es considerable pues hay grupos en los que más del 50\% no cuenta con ellas, lo que podría estar indicando que tal vez se encuentran laborando en la informalidad, en ese sentido se puede incentivar a ese personal incluyéndolos en un sistema de seguridad social.

\section{Referencias}

Bauchet, J., C. Marshall, L.Starita, J. Thomas, y A.Yalouris (2011). Latest Findings from Randomized Evaluations of Microfinance. Washington, D.C. Financial Access Initiative, Innovations for Poverty Action, y Abdul Latif Jameel Poverty Action Lab.

Burgos, B. y López, K. (2010). La situación del mercado laboral de profesionistas. Revista de la Educación Superior, vol. 39. Núm. 156, oct./dic. 2010, México.

Comisión Económica para América Latina y el Caribe (2000). Economic survey of Latin America and the Caribbean 1999-2000. Santiago de Chile.

Cruz, G. (2013). Efecto de la escolaridad sobre el ingreso monetario para 22 ocupaciones en el sector laboral mexicano. Tiempo Económico, vol. VIII, núm. 23, primer cuatrimestre, pp. 37-51, México.

Cruz, G. y Ríos, H. (2014). Elasticidad producto del empleo de los trabajadores en México: un análisis por ocupaciones. Revista Mexicana de Economía y Finanzas, vol. 9, núm. 1, pp. 37-59, México. https://doi.org/10.21919/ remef.v9i1.54

Cull, R., T. Ehrbeck y N. Holle (2014). Financial inclusion and development: recent impact evidence, Washington, DC. World Bank Group, nota de enfoque CGAP; núm. 92.

Fareed, F. et al. (2017), "Financial Inclusion and Women Entrepreneurship : Evidence from

González, G. (2004), La globalización y el mercado de trabajo en México. Revista Problemas del desarrollo, Vol. 35 , núm. 138, julio-septiembre.

Guerrero, C. (2007). Evolución reciente y perspectivas del empleo, México, Naciones Unidas. http://www.cepal.org/ es/publicaciones/5004-evolucion-reciente-perspectivas-empleo-istmo-centroamericano

Instituto Nacional de Estadística y Geografía, (2011). Clasificaciones del Censo de Población y Vivienda 2010. México. http://www.inegi.org.mx/

Instituto Nacional de Estadística y Geografía (INEGI) (2006-2010). Encuesta Nacional de Ingreso y Gasto de los Hogares (ENIGH). México. http://www.inegi.org.mx/

Instituto Nacional de Estadística y Geografía (INEGI) (2009). Encuesta Nacional de Ocupación y Empleo (ENOE). México. http://www.inegi.org.mx/

Instituto Nacional de Estadística y Geografía (INEGI) (1995-2009). Sistema de Cuentas Nacionales de México (SCNM). México. http://www.inegi.org.mx/

Islas, V. (1992). Estructura y desarrollo del sector transporte en México, El Colegio de México, $2^{a}$ edición, México.

Kato, E. (2004). Elasticidad producto del empleo en la industria manufacturera mexicana, Problemas del desarrollo, vol. 35, núm. 138, pp. 87-96, México. http://www.revistas.unam.mx/index.php/pde/article/view/7541

OECD (2017), Economics Department Working Papers, No. 1411, OECD Publishing, Paris. https://doi.org/10.1787/ 2fbd0f35-en

Ríos, J. y Carrillo, S. (2014). El empleo calificado y no calificado en la manufactura de México ante la crisis de 2009, Economía, Sociedad y Territorio, vol. XIV, núm. 46, septiembre-diciembre, pp. 687-714. https://doi.org/10.22136/ est002014393 
Ruiz, C. (2005). Integración de los mercados laborales en América del norte, Cuadernos de Relaciones Laborales, vol. 24, núm. 1, pp. 105-150.

Plan Nacional de Desarrollo 2013-2018 (2013). Programa Sectorial de Comunicaciones y Transportes. México. http:// www.sct.gob.mx/fileadmin/banners/Programa_Sectorial_de_Comunicaciones_y_Transportes.pdf

Perfil de Transporte en México (2014). Instituto Nacional de Estadística y Geografía. México. http://www.inegi.org. $\mathrm{mx} /$

Samaniego, N. (2013). Reseña: Mercado Laboral de Profesionistas en México. Revista Economía UNAM, vol. 10 no. 30, sep/dic 2013, México.

Sydsaeter, K. y Hammond, P. (1995). Matemáticas para el análisis económico. (1ª Ed.) Madrid, Prentice Hall. 